\title{
Uma Análise na Classificação de Risco em Hospitais de Pronto Atendimento
}

\author{
João Paulo Funchal ${ }^{1}$, Cleo Z. Billa ${ }^{1}$, Diana F. Adamatti ${ }^{1}$ \\ ${ }^{1}$ Programa de Pós-Graduação em Computação (PPGComp) \\ Universidade Federal do Rio Grande (FURG) \\ Rio Grande - RS - Brasil \\ \{funchaljoaopaulo, cleobilla, dianaada\}@gmail.com
}

\begin{abstract}
The present paper presents a case study about the use of the mining technique with a risk classification in a public hospital of emergency service. The hospital uses the host standard proposed by the SUS (Brazilian Health System), where it seeks to prioritize the most serious cases to expedite its attendance. The system based on parameters suggest a classification to the patient and a supplier of correspondence classification agree or not with a suggestion and thus can change classification of the patient. This work consists of interpreting the patient classification patterns so that the classification steps can be optimized so that the screening that currently requires four steps can reach a result for the nurse without the need to inform the classification qualifiers by decreasing the time of care and the risks of the patient as well as to make the form of suggestion of the classification closer than the nurse classifies. During the execution of this study it was possible to observe a very great divergence in relation to the suggestion of the system in relation to the classification registered by the nurse. The result found with the rules obtained an accuracy of $80 \%$.
\end{abstract}

Resumo. O presente trabalho apresenta um estudo de caso sobre o uso da técnica de mineração com a classificação de risco em um hospital público de pronto atendimento. Atualmente, o hospital utiliza o padrão de acolhimento proposto pelo (Sistema Único de Saúde), onde busca priorizar os casos mais graves para agilizar seu atendimento. O sistema, com base em parâmetros, sugere uma classificação ao paciente e cabe ao responsável pela classificação concordar ou não com a sugestão, podendo assim mudar a classificação do paciente. $O$ trabalho consiste em interpretar os padrões de classificação dos pacientes, para que assim possam ser otimizados as etapas de classificação, de modo que a triagem, que atualmente necessita de quatro etapas, possa chegar a um resultado ao enfermeiro sem a necessidade de informar os qualificadores da classificação, diminuindo o tempo de atendimento e os riscos do paciente, bem como tornar a forma de sugestão da classificação mais próxima do que o enfermeiro classifica. Durante a execução deste trabalho foi possível observar uma divergência muito grande em relação a sugestão do sistema com relação a classificação registrada pelo enfermeiro. $O$ resultado encontrado com as regras obteve uma acurácia de $80 \%$.

\section{Introdução}

A classificação de risco, ou triagem, tem por objetivo a regulação da demanda do atendimento nos serviços de pronto atendimento, visando identificar o atendimento médico 
imediato e o mediato, organizando assim o fluxo de pacientes que procuram estas unidades [Servin 2014, dos Santos 2014]. Apesar do excesso de demanda que sofrem esse tipo de unidades hospitalares, utilizando deste processo de triagem são minimizados os riscos para os pacientes que as procuram [Albino 2007].

Ao longo dos anos, diversos trabalhos vem abordando o uso de Inteligência Artificial, mais especificamente de sistemas especialistas ou de apoio a decisão, na área de saúde. Ainda assim, estes normalmente focam na questão do diagnóstico, tanto no âmbito da medicina como da enfermagem [Talon 2013, Farias 2013, Teixeira 2012, Sellmer 2013, Lichtenstein 2011, Medeiros 2014, Tenório 2011].

Atualmente, ao ingressar no pronto atendimento do hospital Universitário Dr. Miguel Riet Corrêa Júnior, situado na cidade de Rio Grande, fazendo parte da Universidade Federal do Rio Grande (FURG), o paciente passa por um processo de triagem de risco, no qual, através de um sistema informatizado, o profissional de saúde define qual a prioridade para o seu atendimento. Esse sistema tem por objetivo registrar os dados vitais e sintomas do paciente, deixando totalmente a cargo de quem o acolhe definir a prioridade de atendimento do paciente.

O presente trabalho possui como objetivo principal mapear as regras de classificação de pacientes obtidas através da etapa de mineração de dados, utilizando o algoritmo de árvores de decisão, baseando-se no conhecimento do profissional responsável triagem de risco.

O artigo está estruturado em 4 seções. Na Seção 2 está o referencial teórico necessário para entendimento do trabalho proposto, apresentado também o sistema de classificação de risco disponível no hospital universitário. A Seção 3 realiza a análise dos resultados obtidos pela mineração de dados. $\mathrm{Na}$ Seção 4 estão as conclusões e próximos trabalhos a serem realizados.

\section{Referencial Teórico}

\subsection{Classificação de Risco}

A classificação de risco visa a agilidade no atendimento dos pacientes, de acordo com um protocolo e o grau da necessidade do indivíduo, não somente considerando a ordem de chegada. O sistema de acolhimento com classificação de risco foi desenvolvido na Austrália por volta da década de 90 , onde utilizam cinco níveis de classificação dos pacientes e o tempo de espera é variado [Júnior 2012]. No Brasil, um dos hospitais pioneiros a adotar essa forma de atendimento foi o Hospital Municipal Dr. Mário Gatti, em Campinas/SP, o qual implantou o sistema no ano de 2000, após receber convite do Ministério da Saúde, que possuía como objetivo implantar um programa de humanização nos hospitais brasileiros.

A implantação da classificação de risco em todas as unidades de pronto atendimento é proposta pela portaria 2048/2009 do Ministério da Saúde, onde esse processo de classificação deve ser realizado por profissional de saúde, de nível superior, via de regra um enfermeiro, com treinamento específico e se valendo de protocolos de acolhimento previamente estabelecidos [Ministério da Saúde 2002]. No protocolo proposto pelo SUS (Sistema Único de Saúde), utilizado neste trabalho, mediante o julgamento 
crítico e experiência do enfermeiro, o paciente pode ser classificado nas seguintes cores [dos Santos 2014]:

- VERMELHO: emergência, o atendimento será realizado imediatamente na sala de emergências;

- AMARELO: urgência terá o atendimento priorizado sobre os pacientes classificados como greenVERDE, no consultório ou leito da sala de observação;

- VERDE: sem risco de morte imediato, tendo o atendimento realizado depois que todos os pacientes classificados como VERMELHO e AMARELO forem atendidos;

- AZUL: quadro crônico sem sofrimento agudo ou caso social, deverá ser preferencialmente encaminhado para atendimento em Unidade Básica de Saúde ou atendido pelo Serviço Social.

Por fim, é importante salientar que essa prática não se caracteriza pelo diagnóstico da doença, mas sim pela determinação da prioridade de atendimento, de acordo com a gravidade do paciente [Servin 2014].

\subsection{Sistema de classificação Hospital Universitário - HU}

O presente sistema de classificação de risco foi implantado em Julho de 2012 no Serviço de Pronto Atendimento (SPA) do Hospital Universitário Dr. Miguel Riet Corrêa Júnior da Universidade Federal do Rio Grande (HU/FURG), sendo inicialmente baseado no protocolo de acolhimento do SUS.

Todavia, o sistema permite que os enfermeiros responsáveis pelo serviço de pronto atendimento cadastrem novos sintomas, os relacionem a qualificadores, e por fim definam a sua classificação, permitindo assim que o mesmo se adapte a realidade local. Por exemplo, atualmente o sintoma Diabetes está configurado de acordo com a Tabela 1, ou seja, para cada sintoma relacionado pode-se ter vários qualificadores associados a este sintoma, indicando uma classificação.

Tabela 1. Exemplo de qualificadores e seus classificadores para o sintoma Diabetes

\begin{tabular}{|l|l|l|}
\hline Sintoma & Qualificador & Classificador \\
\hline Diabetes & Sudorese profusa (hipoglicemia) & VERMELHO \\
\hline & $\begin{array}{l}\text { Alteração do estado mental (letargia, confusão men- } \\
\text { tal, agitação, coma) }\end{array}$ & VERMELHO \\
\hline & Dados vitais alterados & VERMELHO \\
\hline & Glicemia $\geq 250 \mathrm{mg} / \mathrm{dl}$ e sinais de desidratação & AMARELO \\
\hline & Dados vitais normais & AMARELO \\
\hline & Glicemia $\geq 250 \mathrm{mg} / \mathrm{dl}$ e assintomático & VERDE \\
\hline & $\begin{array}{l}\text { Glicemia } \leq 250 \mathrm{mg} / \mathrm{dl} \text { e assintomático } \\
\text { História de diabetes e precisando de medicação (re- } \\
\text { ceita) }\end{array}$ & AZUL \\
\hline
\end{tabular}

Neste sistema, a interface de classificação de risco é dividida em quatro etapas distintas, inicialmente o enfermeiro registra os dados básicos do paciente, conforme ilustrado na Figura 1. 


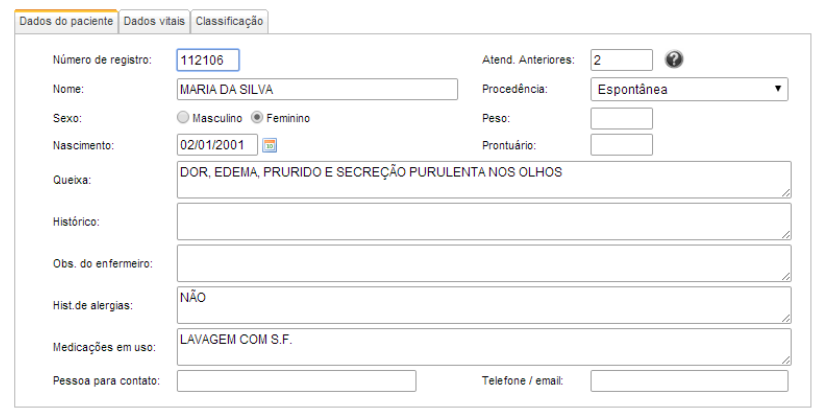

Figura 1. Primeira etapa da classificação, informações básicas

Em seguida, de acordo com a Figura 2, são informados ao sistema os dados vitais do paciente, sua escala de dor e de coma (escala de Glasgow). Na sequência, como

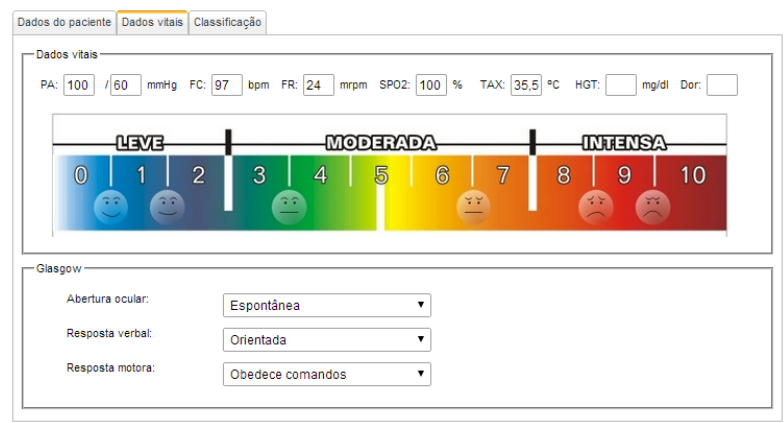

Figura 2. Segunda etapa da classificação, dados vitais

apresentado na Figura 3, o profissional pode escolher os sintomas que ele identifica no paciente (quadro azul da esquerda) e seus respectivos qualificadores (quadro verde da direita). Destaca-se a possibilidade de se marcar diversos sintomas e qualificadores.

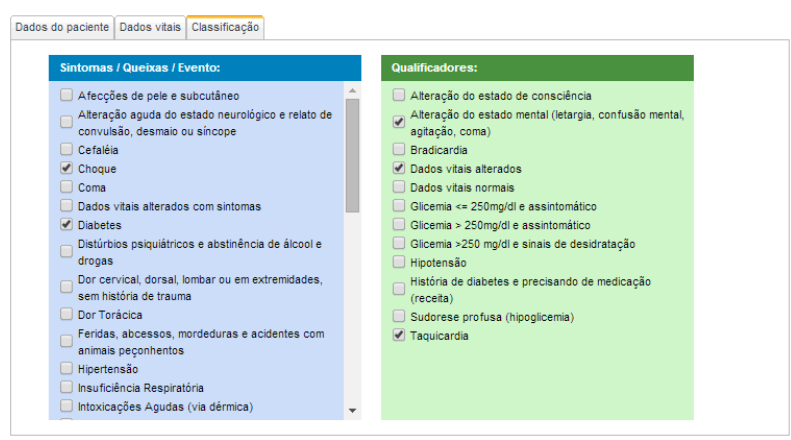

Figura 3. Terceira etapa da classificação, definição de sintomas e qualificadores

Por fim, como ilustrado na Figura 4, o sistema contabiliza o número de classificadores relacionados aos qualificadores selecionados (número entre parênteses) e dado o maior número sugere uma classificação. No entanto, a decisão final fica a cargo do profissional de saúde, que tem a possibilidade de escolher a classificação que ache relevante para o caso. 


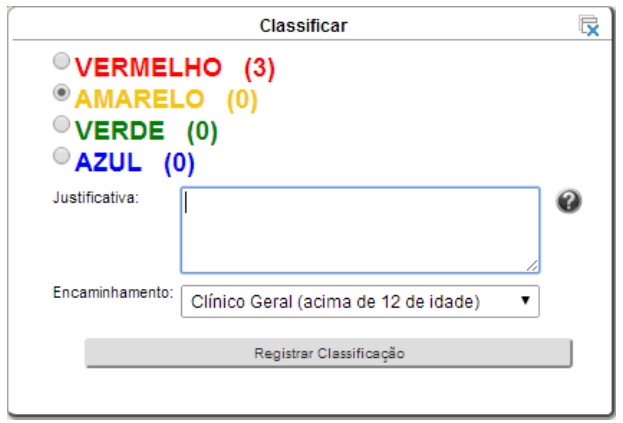

Figura 4. Quarta etapa da classificação, definição do classificador e registro

O sistema de classificação de riscos do HU vem sendo utilizado desde 27/07/2012, com uma média de um atendimento registrado a cada 15 minutos.

A Figura 5 representa a relação de classificação por prioridade, ou seja, o percentual de classificação para cada classificador registrado. Estes dados foram baseados em registros do período 27/07/2012 a 07/02/2017. Pode-se perceber que a classificação com prioridade "VERDE"representa mais da metade dos registros dos pacientes, com 54,6\%. Enquanto a maior prioridade; "VERMELHO", possui um percentual muito abaixo em relação as outras prioridades, ficando em torno de apenas $0,5 \%$.

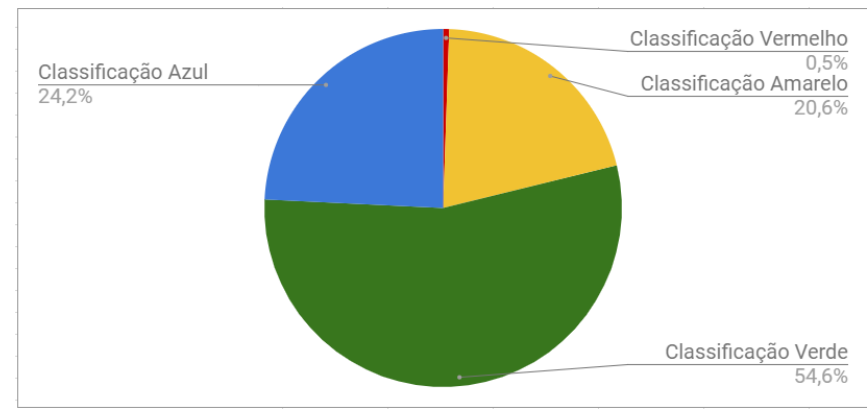

Figura 5. Relação de classificação dos pacientes

Na Figura 6 são apresentados os sintomas informados na classificação do paciente. Nota-se no gráfico que $66,6 \%$ dos casos informados são relacionados aos sintomas de "Queixas abdominais e urinárias" e "Queixas respiratórias", ou seja, mais da metade dos atendimentos estão relacionados apenas dois sintomas.

\subsection{Mineração de Dados}

Segundo [Tan 2009], a mineração de dados é o processo de descoberta automática de informações úteis em grandes depósitos de dados. Suas técnicas agem em expressivas quantidades de bancos de dados para localizar padrões úteis, os quais poderiam ser imperceptíveis. Ainda segundo o autor, há alguns desafios específicos que motivaram a origem e o desenvolvimento da mineração de dados, entre os quais estão: escalabilidade, alta dimensionalidade, dados complexos e heterogêneos, propriedade e distribuição de dados e análises não tradicionais. As tarefas de mineração de dados são divididas em duas categorias, em tarefas de previsão e em tarefas descritivas. Este trabalho, enfocase nas tarefas de previ soa, que também conhecidas como preditivas, tem como objetivo 


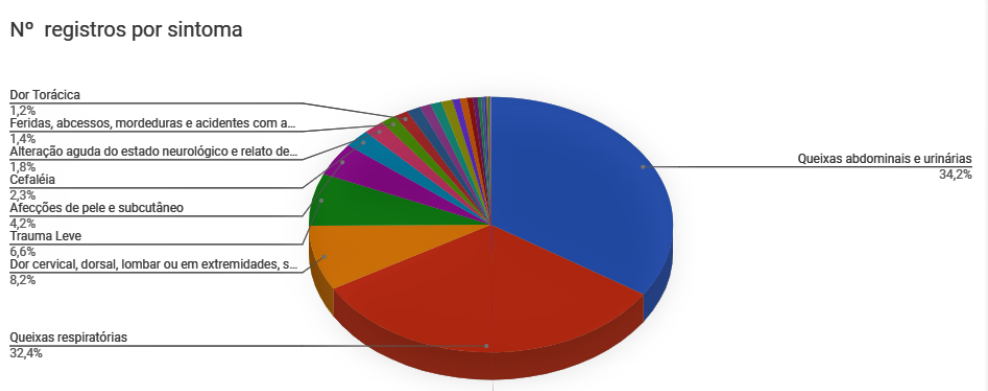

Figura 6. Relação de sintomas registrados

prever o valor de um atributo baseado em valores de outros atributos. O atributo que será previsto é denominado de variável dependente ou alvo, enquanto as variáveis independentes ou explicativas são as denominações usadas para os atributos utilizados para a previsão. Uma das forma de realizar a previsão é baseada na classificação, que abarca inúmeras aplicações e consiste em organizar objetos em categorias pré-definidas. A tarefa de classificação possui dados de entrada que constituem um conjunto de registros, onde cada registro pode ser denominado como instância ou exemplo. Conforme [Tan 2009], são formados por tupla(x,y), no qual o x caracteriza o conjunto de atributos e y o atributo especial, conhecido como rótulo da classificação e atributo alvo ou de categorização. "Classificação é a tarefa de aprender uma função alvo f que mapeie cada conjunto de atributos x para um dos rótulos de classes y pré-determinados"[Tan 2009].

As técnicas de classificação constituem uma sistemática para elaborar modelos de classificação originados de um conjunto de dados de entrada. Podem servir como exemplos, classificadores baseados em regras, redes neurais e classificadores de árvores de decisão, entre outros. E cada técnica utiliza um algoritmo de aprendizagem, o qual é utilizado para construção de modelos que saibam prever rótulos de classes de registros desconhecidos [Tan 2009].

A árvore de decisão é um classificador simples, contudo ainda muito utilizado. Ela possui uma estrutura hierárquica, constituída por nodos e arestas. Um desses nodos é o nodo raiz, no qual não há a presença de arestas de entrada. Além disso, a árvore possui nodos internos, nos quais chega uma aresta e duas ou mais arestas saem. Há também a presença na árvore dos nodos folha ou terminais, que possuem somente uma aresta que chega.

Na técnica de árvore de decisão "cada nodo folha recebe um rótulo de classe. Os nodos não terminais, que incluem o nodo raiz e outros nodos internos, contêm condições de testes de atributos para separar registros que possuam características diferentes"[Tan 2009].

A decisão na árvore de decisão ocorre devido a uma sequência de testes que são executados. Na árvore, cada nó diz respeito a um teste do valor de uma das propriedades, e os valores possíveis do teste correspondem ás ramificações do nó. Segundo [Russell and Norvig 2004], cada nó folha na árvore especifica o valor a ser retornado se aquela folha for alcançada. 
A avaliação do desempenho de um modelo de classificação é baseada nas contagens de registros de testes previstos correta e incorretamente pelo modelo [Tan 2009]. Esta contagem é informada em forma de tabela conhecida como matriz de confusão. Uma matriz de confusão fornece informações necessárias para definir se um modelo de classificação é válido[Tan 2009].

Também através da matriz de confusão, pode-se avaliar outras métricas de avaliação de resultados da classificação, como a precisão e revocação. Sendo a precisão é a parcela de todos os documentos de uma certa classe que realmente à pertencem, em um determinado conjunto de dados [Baeza-Yates and Ribeiro-Neto 2013]. Já a revocação é a parcela de todos os documentos que pertencem a uma determinada classe e que foram corretamente atribuídos a mesma pelo classificador [Baeza-Yates and Ribeiro-Neto 2013].

\subsection{Ferramentas utilizadas}

Foram utilizados para armazenamento e pré-processamento dos dados o Sistema Gerenciador de Banco de Dados (SGBD) PostgreSQL ${ }^{1}$, alguns scripts foram codificados através da linguagem PHP (Hypertext Preprocessor).

Já para a mineração de dados foi utilizada a ferramenta WEKA ${ }^{2}$, que é uma ferramenta que fornece uma interface simples, com uma coleção de algoritmos de diferentes técnicas para etapa de mineração [WEKA 2015]. O WEKA ainda fornece diversas formas de visualizações dos resultados independente da técnica/algoritmo utilizado. Por se tratar de um software livre, pode-se implementar componentes, bem como novos algoritmos na ferramenta.

\section{Análise dos Resultados}

Para a tarefa de mineração executada, foi utilizado o software WEKA [WEKA 2015], onde utilizou-se a técnica de árvores de decisão, mais especificamente o algoritmo J48 [Quinlan 1993]. Importante ressaltar que o atributo alvo definido na configuração do algoritmo J48 é com base na classificação do enfermeiro.

Do conjunto de dados utilizado, buscou-se selecionar os registros classificados pelos principais enfermeiros da classificação de risco entre o período 2012/2017, com um total de 32.828 instâncias. Na configuração do algoritmo J48, utilizou-se para testes o algoritmo de validação cruzadas para validação das regras com 9 grupos para treinamento e 1 grupo para teste (10-fold crossvalidation). O número mínimo de objetos por folha sendo igual a 50. Os atributos da base selecionados foram os quais tiveram sido informados em mais de $60 \%$ dos registros. A Tabela 2 apresenta informações dos atributos utilizados.

Os resultados obtidos foram considerados satisfatórios do ponto de vista geral, como apresenta a Figura 7, com uma acurácia de $80 \%$, taxa de precisão e 79,7\% e revocação de $80 \%$, considera-se bastante expressivo para esses resultados, embora a matriz de confusão, como pode-se notar apresenta alguns valores na diagonal principal classificando como AZUL e VERDE, onde deveriam estar classificados como VERMELHO (maior problema de falso negativo). O ideal da matriz de confusão é estar sempre com a diagonal principal contendo os maiores valores de cada coluna.

\footnotetext{
${ }^{1}$ http: //www.postgresql.org/

${ }^{2}$ http: //www.cs.waikato.ac.nz/ml/weka
} 
Tabela 2. Relação de atributos utilizados para mineração

\begin{tabular}{|c|c|c|}
\hline Atributo & Descrição & Valores apresentados \\
\hline nratendimentosatehmomento & $\begin{array}{l}\text { Apresenta o número de } \\
\text { atendimentos do dia até } \\
\text { o momento em que } \\
\text { o paciente foi classifi- } \\
\text { cado }\end{array}$ & - \\
\hline $\begin{array}{l}\text { nratendimentos- } \\
\text { doenfermeironodia }\end{array}$ & $\begin{array}{l}\text { Apresenta o número } \\
\text { de atendimentos do } \\
\text { enfermeiro no dia an- } \\
\text { terior ao atendimento, } \\
\text { forma para verificar } \\
\text { o volume de atendi- } \\
\text { mento influência na } \\
\text { classificação }\end{array}$ & - \\
\hline turno & $\begin{array}{l}\text { Atributo que identifica } \\
\text { a qual turno o paciente } \\
\text { deu entrada }\end{array}$ & $\begin{array}{l}\{\text { manhã, tarde, noite, madru- } \\
\text { gada }\}\end{array}$ \\
\hline idade & Idade do paciente & {$[0 ; 120]$} \\
\hline cd_sexo & Sexo do paciente & $\{\mathrm{M}, \mathrm{F}\}$ \\
\hline pas & $\begin{array}{l}\text { Pressão } \\
\text { sistólica }\end{array}$ & {$[0 ; 300]$} \\
\hline pad & $\begin{array}{lll}\text { Pressão } & \text { arterial di- } \\
\text { astólica } & & \\
\end{array}$ & {$[0 ; 220]$} \\
\hline fc & Frequência cardíaca & {$[1 ; 210]$} \\
\hline fr & Frequência respiratória & {$[1 ; 80]$} \\
\hline spo2 & $\begin{array}{l}\text { Saturação parcial de } \\
\text { oxigênio }\end{array}$ & {$[8 ; 200]$} \\
\hline glasgow_ocular & Abertura Ocular & {$[1 ; 4]$} \\
\hline glasgow_verbal & Resposta verbal & {$[1 ; 5]$} \\
\hline glasgow_motora & Resposta motora & {$[1 ; 6]$} \\
\hline vl_temp_axila & Temperatura & {$[31 ; 42]$} \\
\hline sintomaid & $\begin{array}{l}\text { Sintomas registrados do } \\
\text { paciente }\end{array}$ & $\begin{array}{l}\text { Sintomas que foram relaci- } \\
\text { onados o paciente, como é } \\
\text { uma relação } 1 \text { x N, ou seja, } \\
1 \text { classificação poderá ter } \mathrm{N} \\
\text { Sintomas, separamos os sin- } \\
\text { tomas em colunas e identi- } \\
\text { ficamos como verdadeiro ou } \\
\text { falso a ocorrência do sintoma }\end{array}$ \\
\hline sintoma_naoinformado & $\begin{array}{l}\text { Verifica se foi regis- } \\
\text { trado algum sintoma }\end{array}$ & $\{$ verdadeiro, falso $\}$ \\
\hline ds_classificador & $\begin{array}{l}\text { Descrição do classifica- } \\
\text { dor registrado }\end{array}$ & $\begin{array}{l}\text { \{Azul, Verde, Amarelo, Ver- } \\
\text { melho }\}\end{array}$ \\
\hline
\end{tabular}




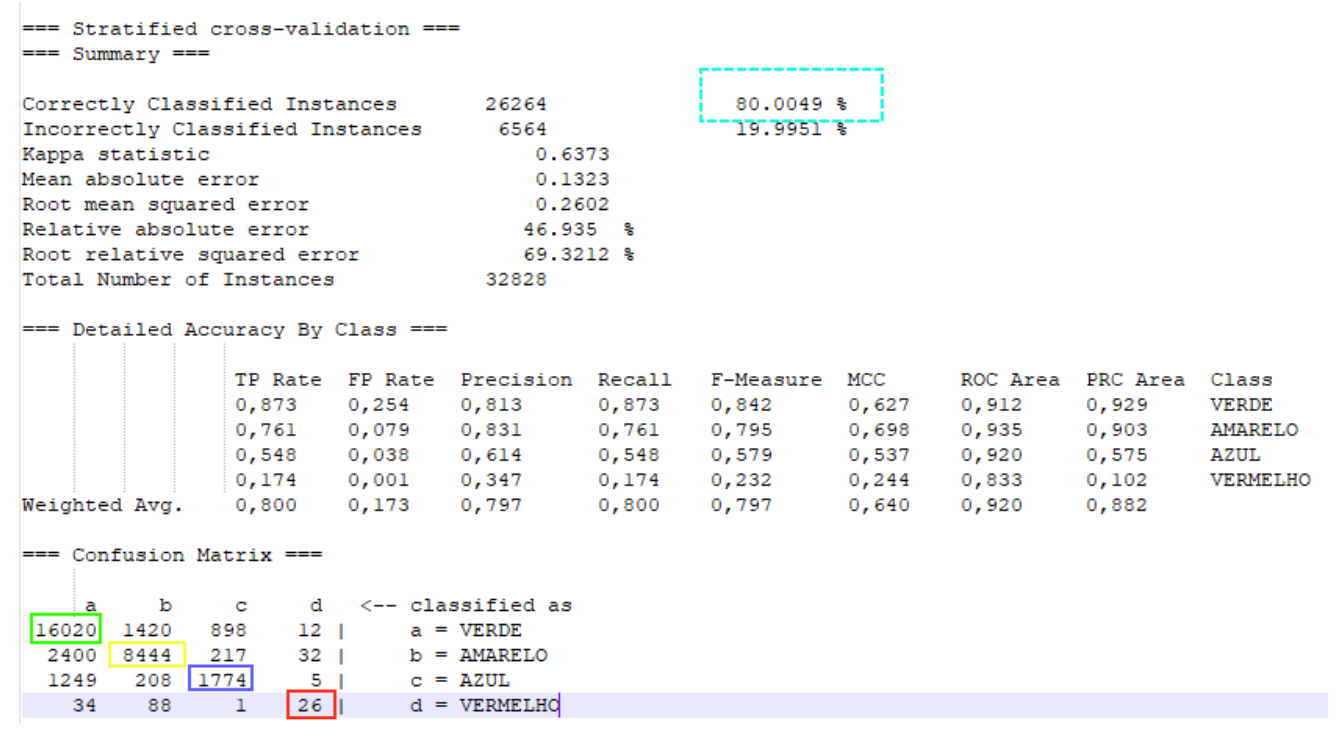

Figura 7. Resultado das regras obtidas

As regras serão apresentadas apenas em pequenos fragmentos na Figura 8, devido a sua extensão. Na Figura 8 (Fragmento 1), pode-se destacar as regras onde o atributo sintomaid_14 não foi informado, ou seja, o paciente não possui o sintoma identificado como 14, saturação parcial de oxigênio superior a 94, sintomaid_20 ocorreu, se o tuno for manhã, o paciente é classificado como VERDE, com uma taxa acerto esta regra de de 96,6\%. Caso o turno seja tarde, o paciente é classificado como VERDE, com uma taxa de acerto em torno de 97,41\%. Na Figura 8 (Fragmento 2), continuação da regra exemplificada na 8 , caso o turno seja noite, idade superior a 12 anos, o número de atendimentos do enfermeiro no dia for superior a 31, o paciente é classificado como VERDE, com uma taxa de acerto de $80,1 \%$, caso contrário serão feitas outras verificações nos atributos.

\section{Conclusões}

A análise da classificação de risco é muito importante para a otimização do sistema de saúde, pois com ela pode-se agilizar os processos, tornar mais justo e mais eficiente o atendimento ao paciente.

Este trabalho apresentou que é possível identificar o comportamento do enfermeiro em algumas situações. Contudo, a matriz de confusão faz repensar o experimento da classificação como um todo, visto que ela tende a errar para os casos mais graves, muito pelo fato de serem o de menor proporção em relação aos outros classificadores (a base é naturalmente desbalanceada, visto que os casos graves são sempre em menor número).

Propõem-se como trabalhos futuros separar os registros por classificadores, criando quatro diferentes estruturas de regras especialistas em cada classificador, para que por fim possa ter segurança na classificação e assim substituir o atual sistema. A partir das regras geradas, implementar no sistema do hospital para que possam servir se apoio aos enfermeiros da classificação de risco. Também visumbra-se o desenvolvimento de um aplicativo móvel, para que profissionais da área da saúde possam utilizar a tecnologia e contribuir para a continuidade do trabalho, para assim melhorar cada vez mais o atendimento dos pacientes que utilizam o serviço de pronto atendimento. 
Outro aspecto bastante importante que pode ser estudanto em classificação de risco é o acompanhamento do paciente após seu ingresso, podendo este evoluir de emergência para urgência, ou seja, existe uma variável tempo em que o paciente é reavaliado para verificar se continua na mesma classificação inicial. Isto pode auxliar bastante no monitoramento dos pacientes, visto que existem casos em que o pacientes e seus acompanhantes não possuem conhecimento o suficiente para saber que o paciente está piorando, ou por medo ou timidez não reclamam pela demora no atendimento.

\section{Referências}

Albino, R. M. (2007). Classificação de risco: Uma necessidade inadiável em um serviço de emergência de qualidade. Arquivos Catarinenses de Medicina, 36(4).

Baeza-Yates, R. and Ribeiro-Neto, B. (2013). Recuperação de Informação - Conceitos e Tecnologia das Máquinas de Busca. Bookman, 2a. edition.

dos Santos, É. A. (2014). Acolhimento com classificação de risco. http://www.pbh.gov.br/smsa/biblioteca/protocolos/ AcolhimentoClassificacaodeRiscodasUpasdeBH.pdf.

Farias, E. P. (2013). Uso de sistema especialista no auxílio ao diagnóstico por meio de escalas de apoio à enfermagem. https: / / repositorio.uninove.br/xmlui/ handle/123456789/525? show=full.

Júnior, J. A. B. (2012). Implantação do sistema acolhimento com classificação e avaliação de risco e uso do fluxograma analisador. Texto \& Contexto - Enfermagem, 21(1).

Lichtenstein, F. (2011). Sistemas de apoio à decisão baseados em diretrizes interpretadas por computador: um breve histórico e outros tópicos. Journal of Health Informatics, $3(4)$.

Medeiros, A. R. C. (2014). Modelo de suporte à decisão aplicado à identificação de indivíduos não aderentes ao tratamento anti-hipertensivo. Saude em debate (SCIELO), 38(100).

Ministério da Saúde (2002). Portaria $\mathrm{n}^{0}$ 2048, de 5 de novembro de 2002. http: //bvsms. saude.gov.br/bvs/saudelegis/gm/2002/prt2048_ 05_11_2002.html.

Quinlan, R. (1993). C4.5: Programs for Machine Learning. Morgan Kaufmann Publishers, San Mateo, CA.

Russell, S. and Norvig, P. (2004). Inteligência artificial. Campus, Rio de Janeiro.

Sellmer, D. (2013). Sistema especialista para apoiar a decisão na terapia tópica de úlceras venosas. Revista Gaúcha de Enfermagem, 34(2).

Servin, S. C. N. (2014). Protocolo de acolhimento com classificaÇÃo de risco. http://bvsms. saude.gov.br/bvs/publicacoes/protocolo_ acolhimento_classificacao_risco.pdf.

Talon, A. F. (2013). Inteligência artificial aplicada à medicina. http://www . fatecbauru.edu.br/ojs/index.php/CET/article/view/76/70.

Tan, P.-N. (2009). Introdução ao data mining. Rio de Janeiro. 
Teixeira, M. J. C. (2012). Os contributos dos sistemas de apoio à tomada de decisão para a prática de enfermagem. Journal of Health Informatics, 4(2).

Tenório, J. M. (2011). Sistemas de apoio a decisões clínicas, inteligência artificial, gastroenterologia. Journal of Health Informatics, 3(1).

WEKA (2015). Weka 3: Data mining software in java. http: / / www . cs . waikato. ac.nz/ml/weka/. 


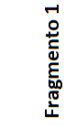

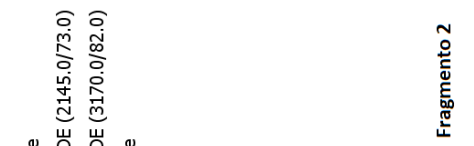

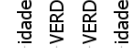

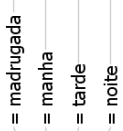

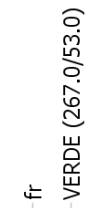

$\frac{\pi}{\sqrt{x}}$

产 을

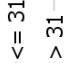

iI III

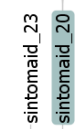

ป

崖

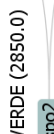

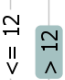

II

ํำ

ํํㅇ

号

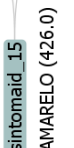

옹 崖猺

III

II

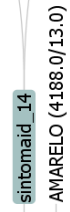

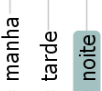

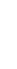

II II II

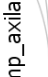

蛋

II II

4

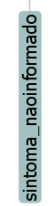

Figura 8. Fragmentos das regras obtidas 